\title{
Special Behaviors in One Pedestrian Flow Experiment
}

\author{
Junlan Chen ${ }^{1 *}$, Wei Wei ${ }^{1} \&$ Jinlian $\mathrm{Wu}^{1}$ \\ ${ }^{1}$ Traffic Engineering, Southeast University, Nanjing, Jiangsu, China \\ *Junlan Chen, E-mail: 1306160554@qq.com
}

Received: July 28, 2017

Accepted: August 8, 2017

Online Published: August 16, 2017

doi:10.22158/wjssr.v4n3p243

URL: http://dx.doi.org/10.22158/wjssr.v4n3p243

\begin{abstract}
We organize one pedestrian flow experiment with 278 participants, and the maximum density reaches 9 $\mathrm{ped} /\left(\mathrm{m}^{\wedge} 2\right)$. The experiment is filmed by one $U A V$, and in the experimental video, we find some interesting behaviors. Five types of these behaviors are classified and introduced: 1) oppression near the boundaries; 2) impact on the boundaries; 3) special moves; 4) absentmindedness; 5) other events. The numbers of Type 1 and 2 behaviors can be counted, while the frequencies of Type 3 and 4 behaviors can be roughly estimated. At one critical density, the results of Type 1, 2, 3, 4 behaviors qualitatively change. This value is about 7 8 ped/( $\left(\mathrm{A}^{\wedge}\right)$, which indicates the possible existence of critical phenomena in pedestrian flow.
\end{abstract}

\section{Keywords}

pedestrian flow, experiment, behavior

\section{Introduction}

The study of pedestrian movement has a very long history, and many models and approaches are used in recent years (Papadimitriou et al., 2009; Ma \& Yarlagadda, 2015). However, in these studies, the main focus is usually on the collective behaviors of many pedestrians, rather than individual behaviors of each pedestrian. We often think the personalities of pedestrians are severely depressed in the crowd, especially when the pedestrian number is large or the density is high. However, in the real life, the features of each pedestrian are always different. Different people have different habits and preferences, so they may choose different ways of walking and exhibit different behaviors. Therefore, when we ignore these individual differences, this oversimplification may lead to some misunderstandings of the essence of pedestrian flow.

In order to investigate the essence of pedestrian flow in a controllable way, some pedestrian flow experiments were conducted in recent years (Daamen \& Hoogendoorn, 2003; Isobe et al., 2004; Helbing et al., 2005; Kretz et al., 2006; Moussaid et al., 2009; Zhang et al., 2011, 2012; Li \& Dong, 2012; Lian et al., 2015). Many of them are recorded by cameras, and the video data can be good resource for the study of pedestrians' behaviors. However, in these papers the individual behaviors of 
pedestrians are seldom studied, which is one common deficiency.

Therefore, we hope to make up for it. We organize one experiment with 278 participants, and the maximum density reaches $p=g_{m}{ }^{-2}$. This experiment is filmed by one Unmanned Aerial Vehicle (UAV), and in the video data we find many interesting behaviors, in which some of them are beyond our previous expectations. These behaviors can be further classified into five types, including 1) oppression near the boundaries, 2) impact on the boundaries, 3) special moves, 4) absentmindedness and 5) other events. The results of Type 1 and 2 behaviors can be counted, while the frequencies of Type 3 and 4 behaviorsonly can be roughly estimated. Then we find the results of Type 1, 2, 3, 4 behaviors qualitatively change at one critical density. This value is about $7 \sim 8 \operatorname{ped} /\left(\mathrm{m}^{\wedge} 2\right)$, which indicates the existence of critical phenomena in pedestrian flow, and needs to be investigated in the future.

This paper is organized as follows. In Section 2 the basic configuration of this experiment is introduced. In Section 3, the microscopic behaviors found in this experiment are presented and classified. In Section 4, the numbers and frequencies of these behaviors are shown and discussed. The final conclusion is given in Section 5 .

\section{The Basic Configuration of the Experiment}

We conducted the pedestrian flow experiment on Dec. 3th, 2016, in the Jiulonghu Campus of Southeast University of China. The experiment was performed on a large square and lasted for about 2.5h with the participation of 278 students. And plastic stools were used to form the boundaries. The radius of the inner circle is $2 \mathrm{~m}$, and that of the outer circle is $3.5 \mathrm{~m}$. So the area of the ring road is about $26 \mathrm{~m}^{2}$. To record the whole experiment, one UAV was used, as shown in Figure 1. During the experiment, it hovers over the center of the two circles, in the height of about $11.8 \mathrm{~m}$.

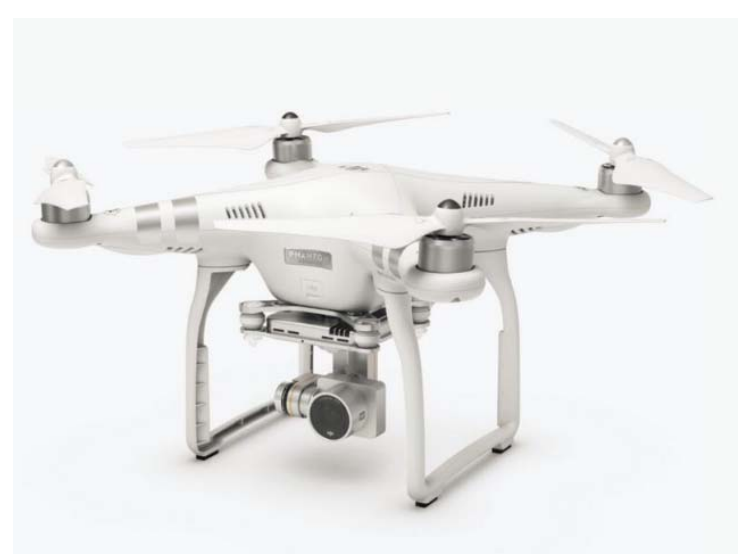

Figure 1. The Unmanned Aerial Vehicle (UAV). It Is the Product of DJI Company

Some rules of the experiment need to be explained. For the high-density conditions, i.e., $p=5,6,7,8$, 
$9 \mathrm{~m}^{-2}$, we make two independent runs (the participants in two runs are not the same ones). For example, if $p=6 \mathrm{~m}^{-2}$, there should be 156 persons on the ring road, and for the bi-directional flow there should be 78 persons in each direction. For the lower density ones, i.e., $\rho=1,2,3,4 \mathrm{~m}^{-2}$, we only make one run, since the situations are very simple and easy to understand. So there are 14 runs through the whole experiment, and the order is: 9-8-7-6-5-9-8-7-6-5-4-3-2-1. For convenience, one specified expression named "predetermined density-order of run-condition" for every single run is used in the following sections. For example, "8-2-U" means the predetermined density is $\rho_{p}=8 \mathrm{~m}^{-2}$, the run is the second run and the condition is uni-directional flow. Similarly, "3-1-B" means the predetermined density is $\rho_{p}=3 \mathrm{~m}^{-2}$, the run is the first run and the condition is bi-directional flow.

The detailed experimental process is described as follows. In each run, half of the participants wear red caps, while the other half wear blue ones. For the convenience of processing individual data, one person wears yellow cap. Firstly we do the experiment for the uni-directional flow, and ask all the participants to move forward. In the first five runs of high densities (9-8-7-6-5), the moving direction is anti-clockwise, and in the next 9 runs it is clockwise. The simple instruction for them is: "walk as usual". When a "steady state" has been reached, we stop them, and immediately start the experiment of bi-directional flow. More specifically, we ask the blue ones and the yellow one to turn around and keep moving forward again, while the red ones remain the previous direction. When another "steady state" is reached again, we end this run, and repeat these steps in the new runs.

When we check the video after the experiment, we find some problems. For example, the actual number of participants in many runs are smaller than we expected, especially under the high density condition. The reason is that there are too many pedestrians in this experiment and we do not have enough time. Therefore, it is impossible to count them before all runs.

The actual numbers of pedestrians in the experiment are shown in Table 1. The "number of total difference" equals the actual total number minus the predetermined one. It is clear that except the first time (9-1), when the density is higher, more pedestrians do not follow the plan and secretly escape. This result is not difficult to understand, since the high-density experiment makes them feel very uncomfortable. Besides, the main reason why there are more participants in the run of 2-1, 3-1 and 4-1 is just the reward: the 4 runs at lower densities belong to the "extra task" of this high-density experiment, and everyone participated can get the overtime pay.

Table 1. The Number of the Pedestrians in the Experiment

\begin{tabular}{|c|c|c|c|c|c|}
\hline Run & $\begin{array}{l}\text { Red number } \\
\text { (Predetermined/Actual) }\end{array}$ & $\begin{array}{l}\text { Blue number } \\
\text { (Predetermined/Actual) }\end{array}$ & $\begin{array}{l}\text { Total number } \\
\text { (Predetermined/Actual) }\end{array}$ & $\begin{array}{l}\text { Number of total } \\
\text { difference }\end{array}$ & $\begin{array}{l}\text { Actual density } \\
\left(\mathrm{m}^{\mathbf{- 1}}\right)\end{array}$ \\
\hline $9-1$ & $117 / 117$ & 116/117 & $234 / 235$ & 1 & 9.04 \\
\hline 8-1 & $104 / 100$ & 103/99 & $208 / 200$ & -8 & 7.69 \\
\hline $7-1$ & $91 / 78$ & $90 / 84$ & $182 / 163$ & -19 & 6.27 \\
\hline
\end{tabular}




\begin{tabular}{llllll}
\hline $6-1$ & $78 / 70$ & $77 / 76$ & $156 / 147$ & -8 & 5.65 \\
$5-1$ & $65 / 65$ & $64 / 60$ & $130 / 126$ & -4 & 4.85 \\
$9-2$ & $117 / 111$ & $116 / 105$ & $233 / 217$ & -16 & 8.35 \\
$8-2$ & $104 / 99$ & $103 / 99$ & $208 / 199$ & -9 & 7.65 \\
$7-2$ & $91 / 83$ & $90 / 83$ & $182 / 167$ & -15 & 6.43 \\
$6-2$ & $78 / 74$ & $77 / 73$ & $156 / 148$ & 5.69 \\
$5-2$ & $65 / 65$ & $64 / 57$ & $130 / 123$ & -8 & 4.73 \\
$4-1$ & $52 / 52$ & $51 / 52$ & $104 / 105$ & -7 & 4.04 \\
$3-1$ & $39 / 42$ & $38 / 40$ & $78 / 83$ & 1 & 3.19 \\
$2-1$ & $26 / 28$ & $25 / 27$ & $52 / 56$ & 5 & 2.15 \\
$1-1$ & $13 / 13$ & $12 / 12$ & $26 / 26$ & 4 & 1.00 \\
\hline
\end{tabular}

Besides, there are other problems which have some negative influence on the extraction of pedestrians' velocities and trajectories, including the random motion of the UAV, and the inevitable errors when we measure the positions of pedestrians, etc. However, it has no influence on the observation of the behaviors discussed in this paper, and the statistics still can be accurate. And the discussion about the pedestrian dynamics will be presented in some other papers.

\section{The Classification of Microscopic Behaviors}

The video data of this experiment show us lots of interesting microscopic behaviors, especially when $\rho_{p} \geq 5 m^{-2}$. We think it is necessary to study the mechanisms of these behaviors, which are rarely found under low-density conditions, neither in our data nor some other previous data. In a nut shell, the behaviors occurred in the experiment can be classified into five types:

\subsection{Oppression near the Boundaries}

When density is high, the pedestrians may suffer very bad experiences, and their movement may be seriously affected by the boundaries. For example, one or several adjacent pedestrians simultaneously stumble and fall (see Figures 2, 3). What's more, one can sit on the stools for a while (see Figure 4), and even temporarily jump out of the circles (see Figure 5) in order to avoid the serious congestions. 


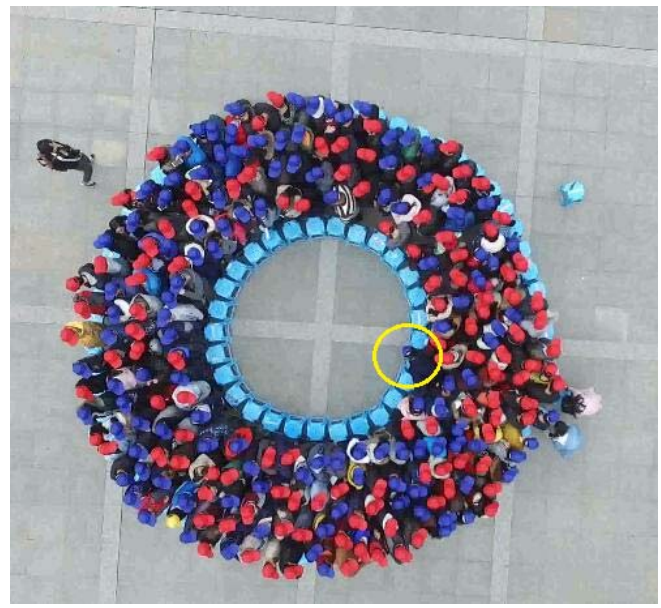

Figure 2. One Pedestrian Stumbles and Falls, in 9-1-U

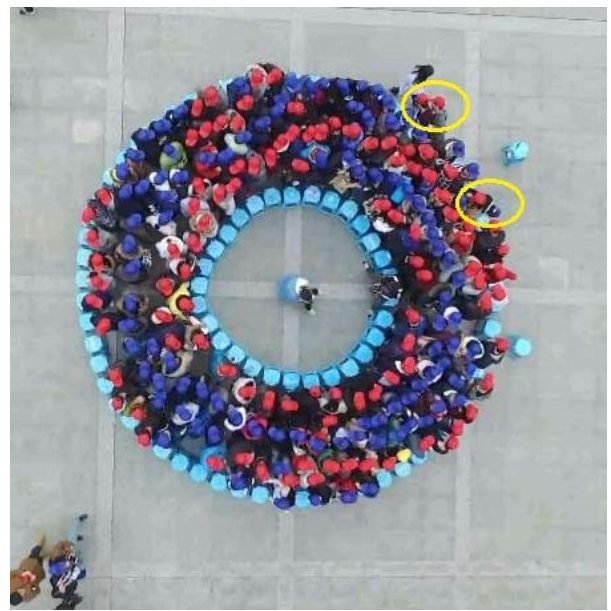

Figrue 3. Several Adjacentpedestrians Stumble and Fall Simultaneously, in 8-1-B

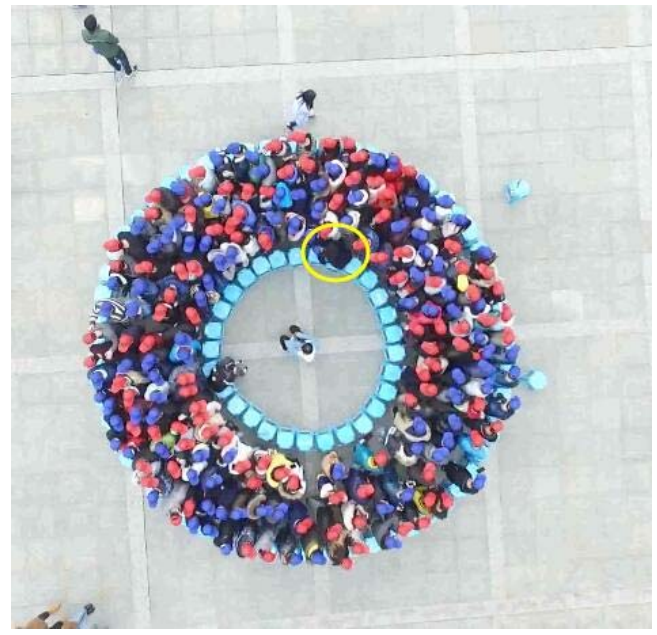

Figure 4. One Pedestrian Sits on the Stools for a While, in 8-1-U 


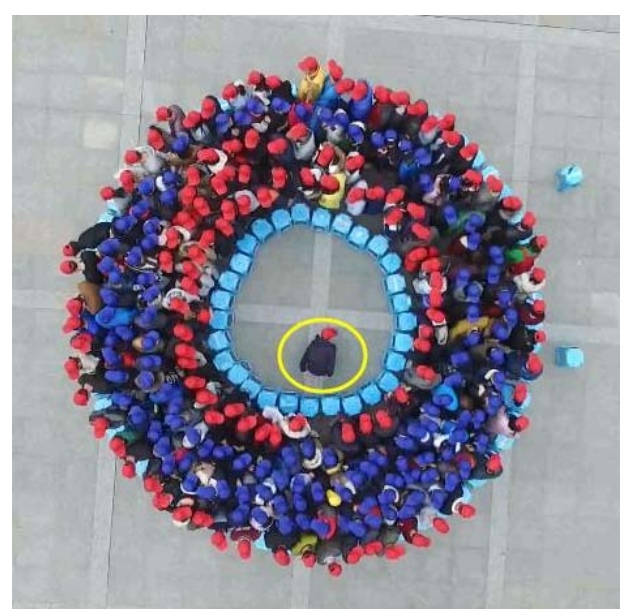

Figure 5. One Pedestrian Temporarily Jumps out of the Circles, in 9-1-B

\subsection{Impact on the Boundaries}

It is easy to understand that when density is high, the two boundaries may be also shocked by the pedestrians. Since the plastic stools used in the experiment are temporarily tied by sellotape, they are not so strong. Sometimes the staffs need to arrange them, in order to prevent the boundaries from being damaged, as shown in Figures 6, 7. Sometimes we forget to let any staff stay in the inner circle, and we can observe that the boundaries are out of shape due to the frequent collisions, as shown in Figure 8.

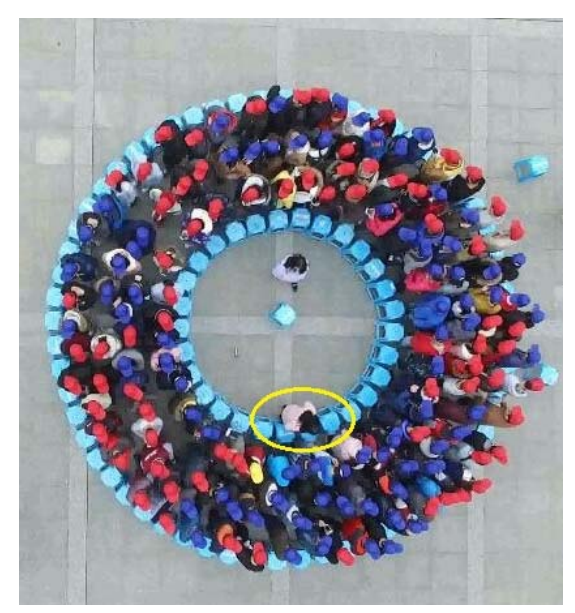

Figure 6. Type 2 Behaviors: Impact on the Boundaries: One Staff Arranges the Stools, in 6-1-U 


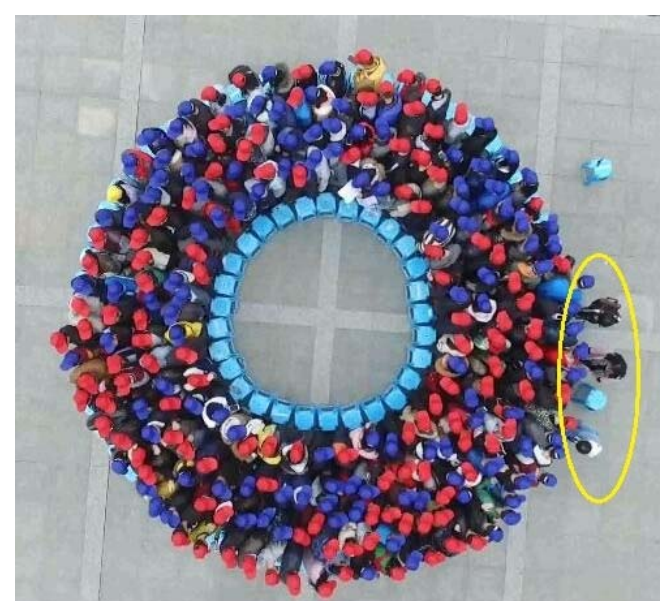

Figure 7. Type 2 Behaviors: Impact on the Boundaries: Three Staffs Arrange the Stools at the Same Time, in 9-1-U

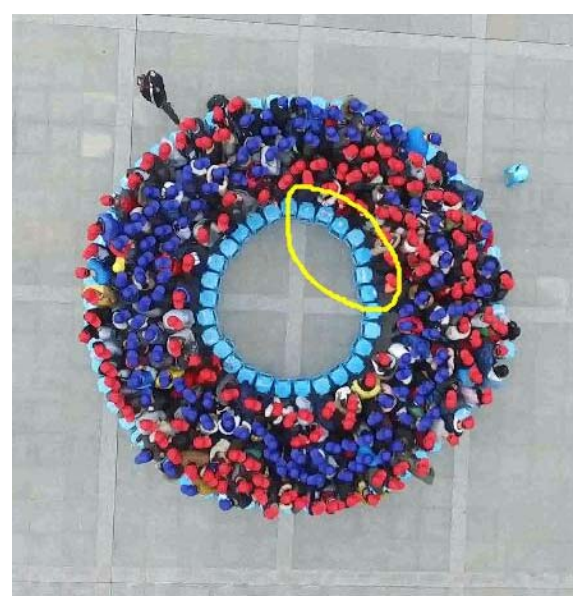

Figure 8. Type 2 Behaviors: Impact on the Boundaries: The Boundaries Are out of Shape When There Is no Staff Inside the Circle, in 9-1-B

\subsection{Special Moves}

During the experiment, sometimes pedestrians choose some special way to move, rather than normally and regularly walking forward. At least there are two tendencies:

1) To be similar to others. For example, many pairs are automatically formed, and both of them can move together, as shown in Figure 9. Sometimes more people are involved and large clusters can be formed: they hug each other, as shown in Figure 10. Maybe they have known some others before the experiments (e.g., they belong to the same class).

2) To be different from others. For example, when there exists a large gap at some medium densities, hestarts running, as shown in Figure 11. On the contrary, sometimes when the gap is not large enough, there is still someone who wants to move forward as quickly as possible, as shown in Figure 12. Here 
we also find some features of sexuality, since most behaviors of this subtype belong to male pedestrians.

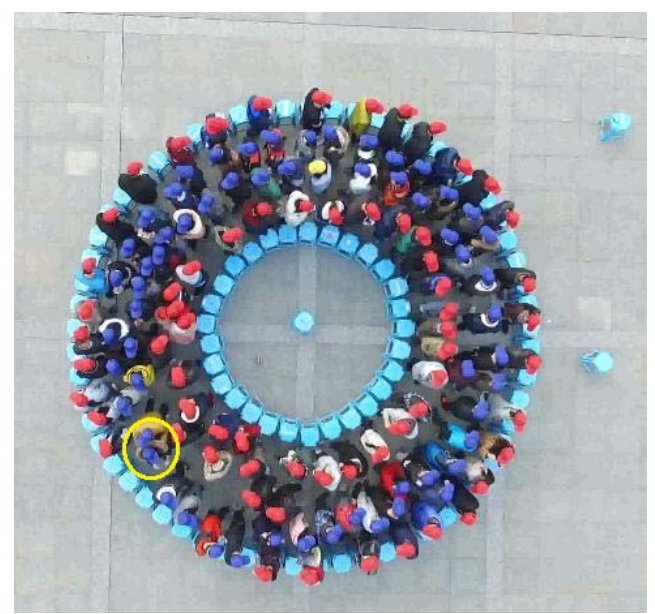

Figrue 9. Type 3 Behaviors: Special Moves: One Pedestrian Arms the Waist of the Front One and Move Together, in 5-2-B

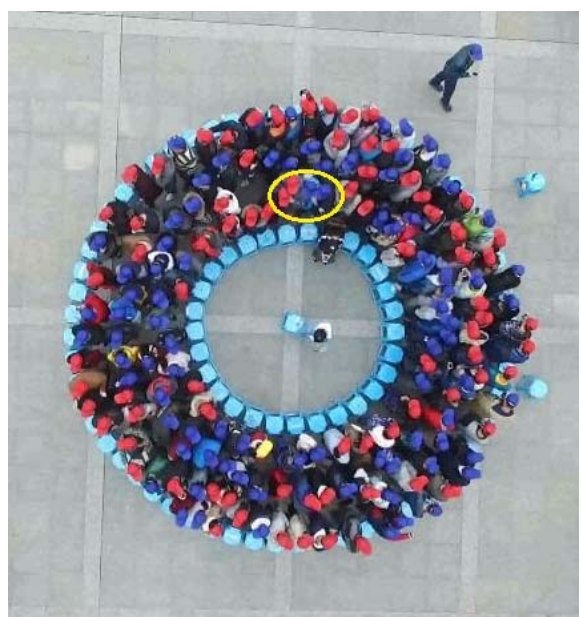

Figure 10. Type 3 Behaviors: Special Moves: Four Pedestrians Hug Each Other and Move Together, in 7-1-U 


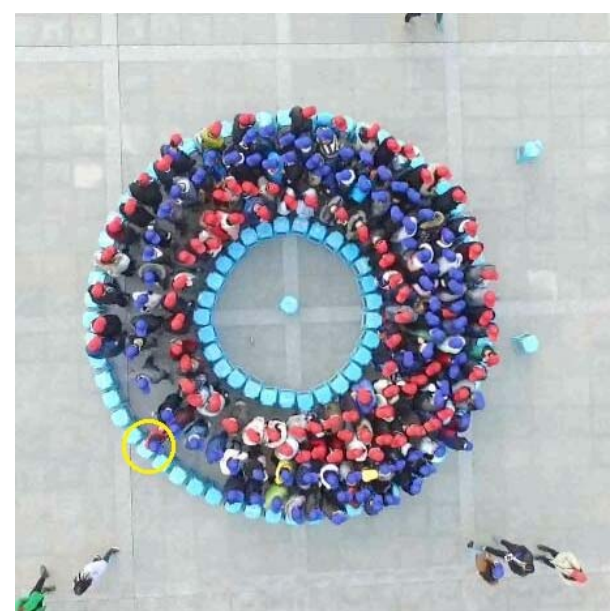

Figure 11. Type 3 Behaviors: Special Moves: One Pedestrian Starts to Run When the Gap Is Large, in 7-2-B

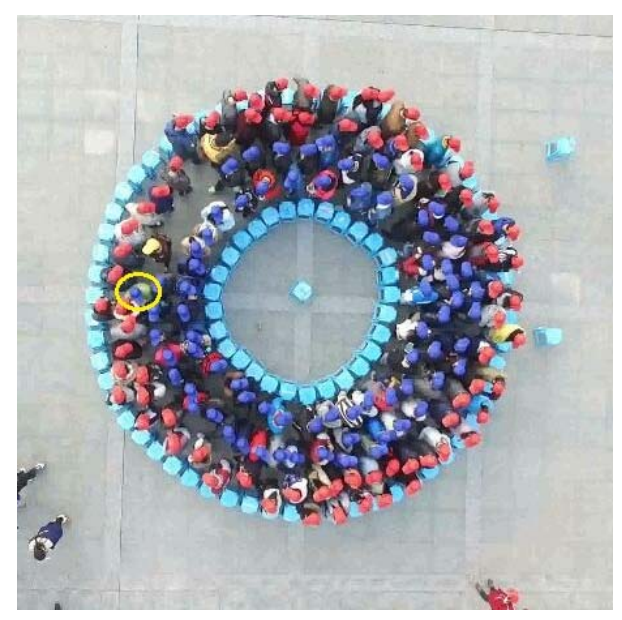

Figure 12. Type 3 Behaviors: Special Moves: One Pedestrian Tries to Press Forward When the Gap is Small, in 6-2-B

\subsection{Absentmindedness}

When the space is large, it is possible for the pedestrians to do something else, such as talking to some other people (e.g., the staff in Figure 13, the passer-by in Figure 14), reading some materials for the incoming exams (see Figure 15), taking off the caps temporarily (see Figure 16), etc. The pedestrians often talk to each others, but it is difficult for the UAV to capture these details, especially when density is high. In addition, the phenomenon of "pedestrian playing with mobile phones" are very common during the experiment, as shown in Figure 17. 


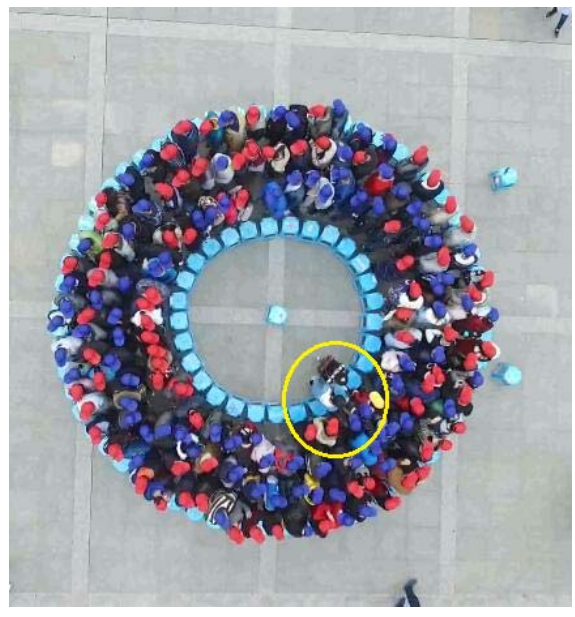

Figure 13. Type 4 Behaviors: Absentmindedness: Five Pedestrians (Including the Yellow One) Talk to the Staff, in 7-1-U

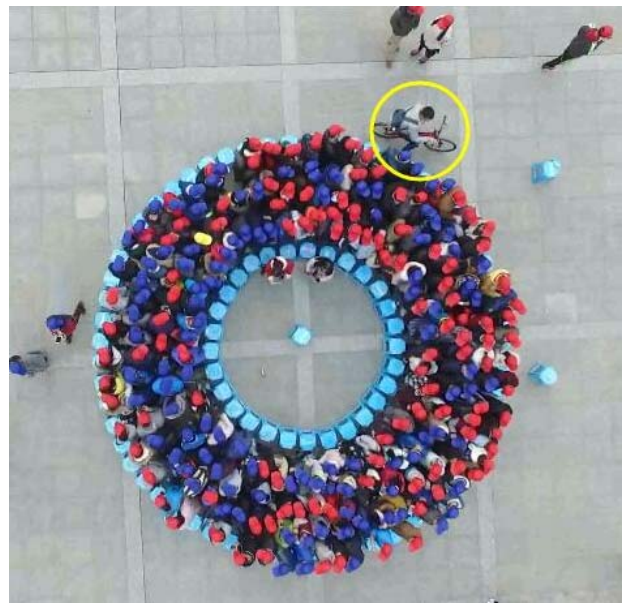

Figrue 14. Type 4 Behaviors: Absentmindedness: Two Pedestrians Talk to One Passer-by on the Bicycle, in 9-2-U

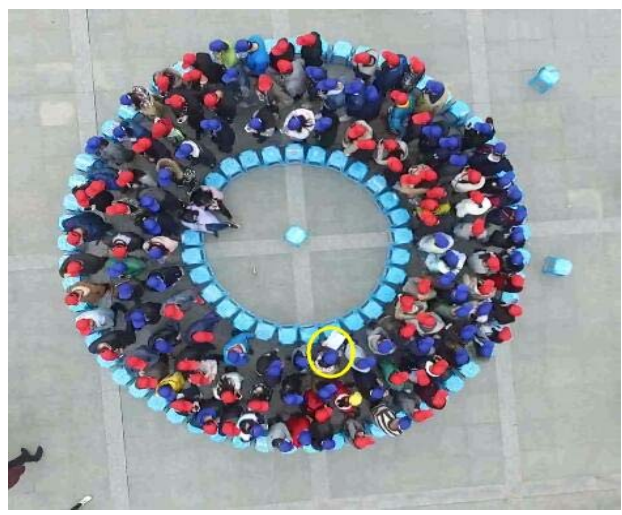

Figrue 15. Type 4 Behaviors: Absentmindedness: One Pedestrian Reads Some Materials, in 5-1-U 


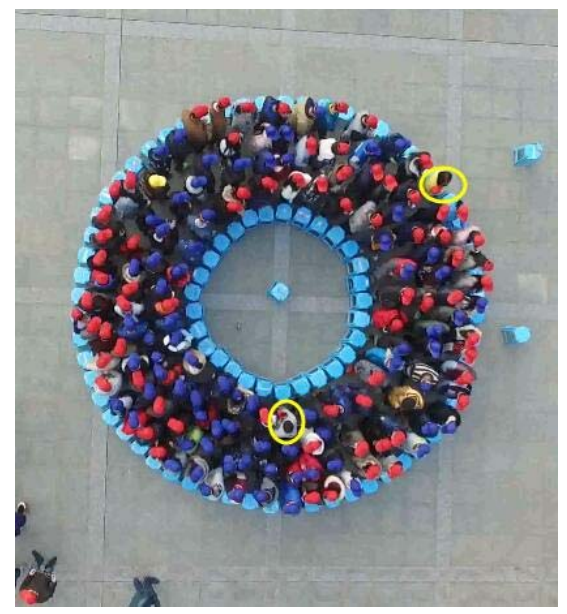

Figrue 16. Type 4 Behaviors: Absentmindedness: Two Pedestrians Take off the Caps Temporarily, in 6-2-U

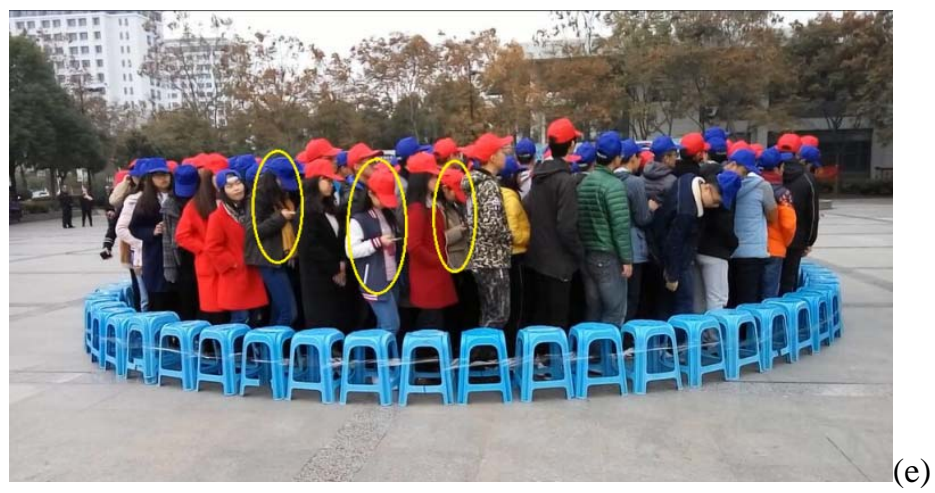

Figure 17. Type 4 Behaviors: Absentmindedness: Three Pedestrians Play with Mobile Phones, in 7-1-B

\subsection{E: Other Events}

In our experiment, there are some phenomena which do not belong to anyone of Type 1, 2, 3, 4 behaviors. For example in Figure 18, we can see two pedestrians lay down on the stools and slowly pass. It is easy to understand, since there are not enough room left, and this is the only way they can go through. However, this behavior does not belong to Type 1, since the pedestrians are not being oppressed. At the same time, it also does not belong to Type 3, since they do not have other choices and they must do it. Thus we category it into Type 5.

On the other hand, there are some other very rare events, which are beyond our expectations. Many of them are not immediately noted during the experiment, and only found in the video. For example, as shown in Figure 19. we can see some surprising scene in 9-1-B: two pedestrians are exchanging their caps and identities. And then, they can move and form pedestrian lanes more easily. Of course, this behavior breaks the rules of the experiment, but it still has some meaning: in order to escape the 
disordered situation, people can cooperate with others, and their "intelligence" is always very strong. Besides, in some videos, we find one pedestrian always wears one black cap which belongs to himself, as shown in Figure 20. This special behavior expresses some complex emotions to the experiment, which is not easy to analyze. At least, it is a trick against the rules.

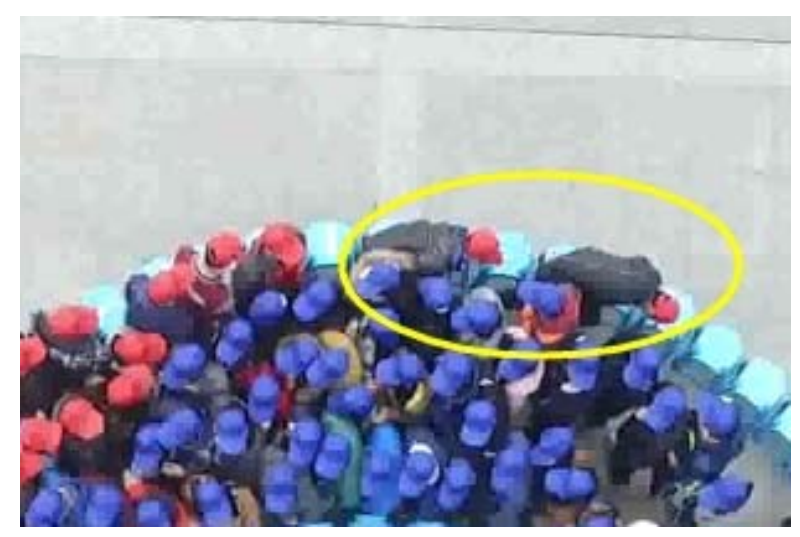

Figrue 18. Type 5 Behaviors: Other Events: the Pedestrians' Movement When the Space Is Narrow, in 8-2-B

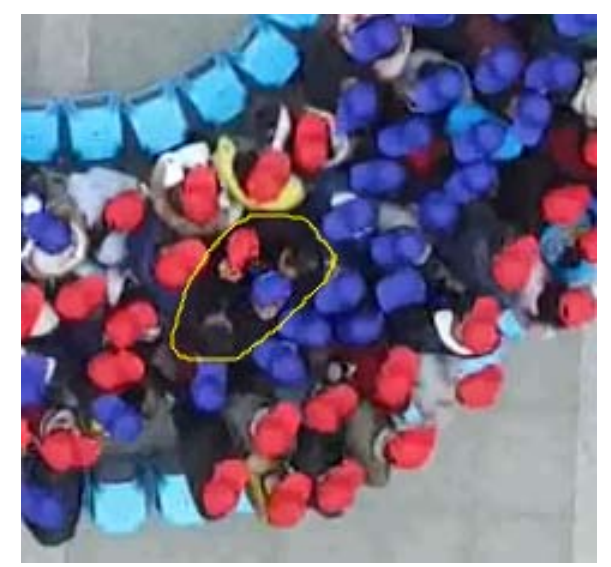

Figrue 19. Type 5 Behaviors: Other Events: the Exchange of Caps and Identities, in 9-1-B 


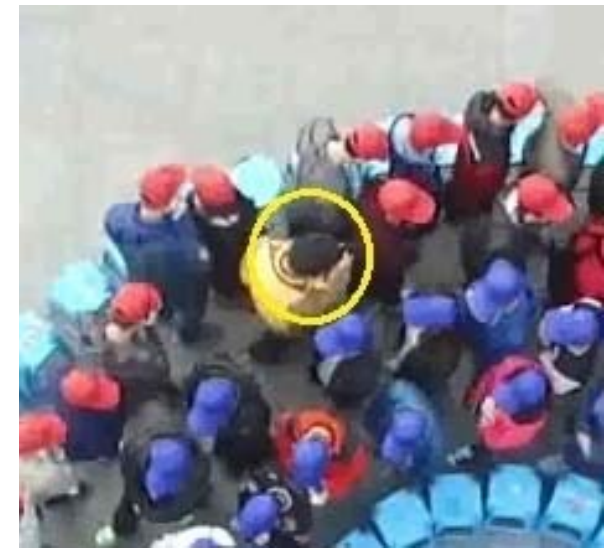

Figrue 20. Type 5 Behaviors: Other Events: the Black Cap, in 6-2-B

\section{The Statistical Results of Microscopic Behaviors}

The properties of the microscopic behaviors found in the experiment are different. For example, there are not so many Type 1, 2 or 5 behaviors, and their numbers can be count and presented. On the contrary, it is impossible to accurately measure the results of Type 3 or 4 behaviors: for example, how long does one pedestrian play with mobile phone cannot be clearly identified in the UAV video. Therefore, we only make some rough estimations. We use two phrases to describe the frequencies of Type 3 and 4 behaviors: "few" and "some”. For Type 4 behaviors, we also use "many", since there are obvious differences between the three results.

Table 2. The Special Behaviors Under High Densities

\begin{tabular}{lllllll}
\hline Run & Actual & Time duration & 1. Oppression near & 2. Impact on & 3. Special & 4. Absent-mindedness \\
& density $\left(\mathbf{m}^{-2}\right)$ & $(\mathrm{mm}: \mathrm{ss})$ & the boundaries & the boundaries & moves & \\
\hline 9-1-U & 9.04 & $7: 40$ & 2 & 6 & Few & Some \\
8-1-U & 7.69 & $4: 28$ & 4 & 6 & Few & Few \\
7-1-U & 6.27 & $4: 39$ & 0 & 2 & Some & Some \\
6-1-U & 5.65 & $2: 32$ & 0 & 2 & Some & Some \\
5-1-U & 4.85 & $2: 00$ & 0 & 2 & Some & Many \\
9-2-U & 8.35 & $3: 51$ & 1 & 1 & Few & Few \\
8-2-U & 7.65 & $2: 31$ & 0 & 3 & Few & Few \\
7-2-U & 6.43 & $2: 19$ & 0 & 1 & Few & Some \\
6-2-U & 5.69 & $1: 50$ & 0 & 0 & Some & Some \\
5-2-U & 4.73 & $1: 48$ & 0 & 0 & Some & Many \\
9-1-B & 9.04 & $9: 40$ & 7 & 3 & Few & Few \\
8-1-B & 7.69 & $5: 05$ & 6 & 3 & Few & Few \\
$7-1-B$ & 6.27 & $4: 11$ & 3 & Some & Some \\
\hline
\end{tabular}




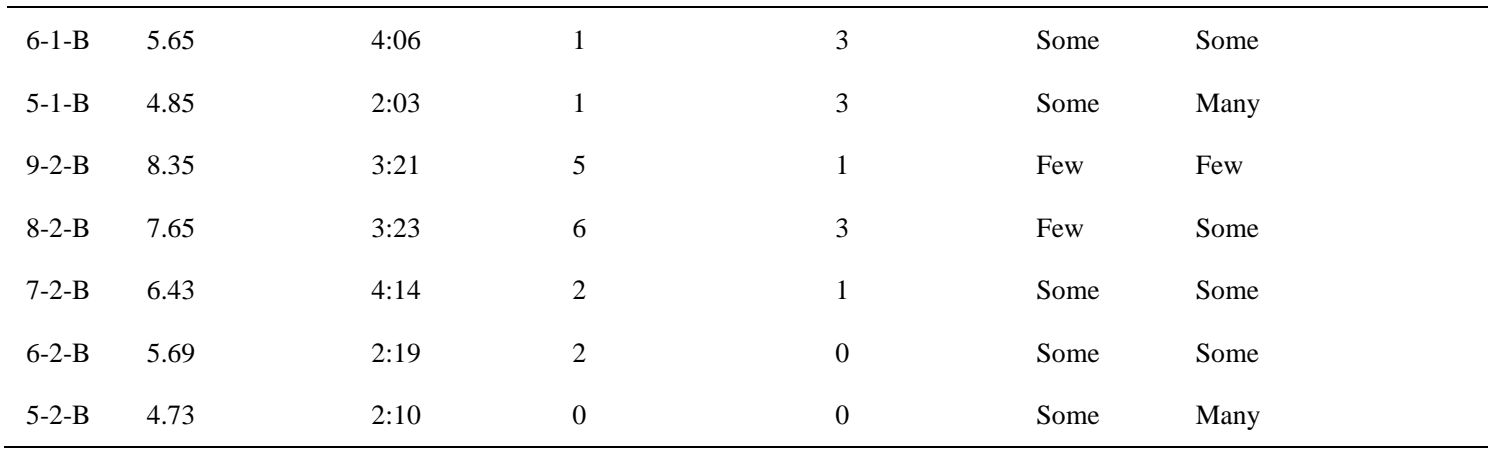

The statistical results of these behaviors are shown in Table 2, and some laws can be found:

(1) The differences between uni-directional flow and bi-directional flow are obvious: the number of Type 2 behaviors in uni-directional flow is larger, while the number of Type 1behaviors in bi-directional flow is larger. We think it is due to the fact that for uni-directional flow, all the pedestrians can be considered as one integrated whole, so it can make some impacts on the surrounding environment. But for bi-directional flow, the conflicts between two groups of pedestrians are obvious, so the forces cannot be concentrated.

Besides, from the observation of videos, it seems that the frequency of Type 3 behaviors in bi-directional flow is a little higher, and the frequency of Type 4 behaviors in uni-directional flow is a little higher. For example, most subfigures in Figure 4 come from the bi-directional flow, while most subfigures in Figure 5 come from uni-directional flow. This tendency is reasonable, since in uni-directional flow the pedestrians only need to follow all the others, while in bi-directional flow they have to find the paths by themselves. But these differences are not so obvious and difficult to measure, thus we do not present them in the table.

(2) The differences between two runs are also obvious, but it is mainly due to the simple fact that pedestrians quickly get familiar with the basic configuration of the experiment. Thus these pedestrians can reach one steady state with shorter time. The numbers of Type 1 and Type 2 behaviors also decrease, which implies they know how to avoid the conflicts with the boundaries. Besides, we find the effect of different initial moving directions (anti-clockwise and clockwise) is not obvious in all the results.

(3) Another important result is about the criticality of densities. The special results in Table 2 are marked by the color of red, including the higher results in the columns of Type 1 and 2, and the results of "few" in the columns of Type 3 and 4. It is clear that all the higher values of Type 1 and 2behaviors, and most of the lower values of Type 3 and 4behaviors can be found in the results of 9-1, 9-2, 8-1 and 8-2, which means these 4 runs are different from the others. Besides, the reason why the results of Type 4behaviors in 9-1-U and 8-2-B are "some", is the long-time stop of all the pedestrians during the experiment. Actually, in 9-1-U one mega jam is found, and in 8-2-B one typical deadlock emerges. Both of them belong to the special situations in the experiment, which are also different from the other 
10 runs with lower densities.

Therefore, we think there exists one critical density at about $p_{\mathrm{c}}=7 \mathrm{~m}^{-2}$. The results of microscopic behaviors when $\rho \geq 7.6 \mathrm{~m}^{-2}$ are qualitatively different from that when $\rho \leq 6.5 \mathrm{~m}^{-2}$, and the two main differences are:

(1) The phenomena near boundaries. Above this critical density, the oppression from boundaries and the impact on boundaries are significant, while below this critical density, they are not.

(2) The freedom and experience of individuals. For the pedestrians, all these experiments are boring. But above this critical density, they have to mainly focus on the surrounding environment and their own safety; while below this value, they have more choices: they can do something else as they want, and the individualities emerge.

Finally, we can recall the important traffic phenomena named breakdown, which means the sudden drop of some parameters within a short range. For example, the breakdown in vehicular traffic flow is often observed in the fundamental diagrams of many empirical data (Helbing, 2001; Kerner, 2004), in which the averaged flow and averaged velocity drastically drop in some density range. This phenomenon is widely recognized in this field, and different explanations can be given. However, whether it can be found in pedestrian flow is not clear. We think the behaviors discussed in this paper may indicate the breakdown phenomena of pedestrian flow, but we still need more data to check this hypothesis in the future. For example, we need to find some ways to quantitatively measure the factors of pedestrians' comfortableness, and then, build one new system for better explanations about the stochasticityand the complexity in the pedestrian flow.

\section{Conclusion}

In this paper we discuss the special behaviors of some pedestrians in one experiment. Five types of behaviors are introduced and classified, including 1) oppression near the boundaries, 2) impact on the boundaries, 3) special moves, 4) absentmindedness and 5) other events. The numbers of Type 1 and 2 behaviors, and the frequencies of Type 3 and 4 behaviors qualitatively change at one critical density $\rho_{\mathrm{c}}=7 \sim 8 m^{-2}$. This critical density is very important for pedestrian flow, which implies the change of the experience of individuals and the possible existence of breakdown phenomena. We think it should be further investigated in the following studies.

Although this paper is a good beginning, some problems still exist. For example, many of the data used in this paper are only rough estimations, since the numbers of various behaviors (especially Type 3 and 4 Behaviors) are difficult to measure. The qualitative results make it difficult to study the functions between different variables. Besides, since the main purpose of this experiment is to study the pedestrian flow dynamics under periodic boundary conditions, we do not plan to make a survey on the participants, which is a little pity. Of course, for the study of pedestrian psychology, making a survey is 
very important and necessary, which can be conducted in the future experiments. The questionnaires can be collected shortly after the experiment, and just before they get the reward. We believe the quantitative data can be more useful for the further discussion and modeling.

\section{Acknowledgements}

We thank all the participants and all the staffs in this large-scale experiment, which is very difficult to organize. We also thank Cheng-Jie Jin for useful discussions. This work is funded by the Natural Science Foundation of Jiangsu Province (BK20150619).

\section{References}

Daamen, W., \& Hoogendoorn, S. P. (2003). Controlled experiments to derive walking behaviour. European Journal of Transport and Infrastructure Research, 3, 39-59.

Helbing, D. (2001). Traffic and related self-driven many-particle systems. Rev. Mod. Phys, 73, 1067-1141. https://doi.org/10.1103/RevModPhys.73.1067

Helbing, D., Buzna, L., Johansson, A., \& Werner, T. (2005). Self-organized pedestrian crowd dynamics: Experiments, simulations and design solutions. Transportation Science, 39, 1-24. https://doi.org/10.1287/trsc.1040.0108

Isobe, M., Adachi, T., \& Nagatani, T. (2004). Experiment and simulation of pedestrian counter flow. Physica A, 336, 638-650. https://doi.org/10.1016/j.physa.2004.01.043

Kerner, B. S. (2004). The Physics of Traffic. Springer, Heidelberg. https://doi.org/10.1007/978-3-540-40986-1

Kretz, T. et al. (2006). Experimental study of pedestrian counter flow in a corridor. Journal of Statistical Mechanics, 10001. https://doi.org/10.1088/1742-5468/2006/10/P10001

Li, X., \& Dong, L. Y. (2012). Modeling and Simulation of Pedestrian Counter Flow on a Crosswalk. Chinese Physics Letters, 29, 098902. https://doi.org/10.1088/0256-307X/29/9/098902

Lian, L. et al. (2015). An experimental study on four-directional intersecting pedestrian flows. Journal of Statistical Mechanics, 08024. https://doi.org/10.1088/1742-5468/2015/08/P08024

Ma, W., \& Yarlagadda, P. K. D. V. (2015). Pedestrian Dynamics in Real and Simulated World. Journal of Urban Planning and Development, 141, 04014030. https://doi.org/10.1061/(ASCE)UP.1943-5444.0000232

Moussaid, M. et al. (2009). Experimental study of the behavioural mechanisms underlying self-organization in human crowds. Proceedings of the Royal Society B-Biological Sciences, 276, 2755-2762. https://doi.org/10.1098/rspb.2009.0405

Papadimitriou, E., Yannis, G., \& Golias, J. (2009). A critical assessment of pedestrian behaviour models. Transportation Research Part F, 12, 242-255. https://doi.org/10.1016/j.trf.2008.12.004

Zhang, J., Klingsch, W., Schadschneider, A., \& Seyfried, A. (2011). Transitions in pedestrian 
fundamental diagrams of straight corridors and t-junctions. Journalof Statistical Mechanics, 06004.

Zhang, J., Klingsch, W., Schadschneider, A., \& Seyfried, A. (2012). Ordering in bidirectional pedestrian flows and its influence on the fundamental diagram. Journal of Statistical Mechanics, 02002. https://doi.org/10.1088/1742-5468/2012/02/P02002 